\title{
Computational Analysis of the Effect of Bogie Inclination Angle on Landing Gear Noise
}

\author{
K.J. van Mierlo, K. Takeda ${ }^{\dagger}$ and E. Peers ${ }^{\ddagger}$ \\ University of Southampton, Southampton, UK
}

\begin{abstract}
Airframe noise and in particular main landing gear noise is a major noise source during the approach phase. Wind tunnel tests have shown a strong relationship between the inclination angle of the bogie and the noise radiation of a main landing gear. Using Computational Fluid Dynamics, this paper investigates the flow features around three different configurations of a simplified four wheel main landing gear. The three configurations consist of a horizontal, $10^{\circ}$ toe up and $10^{\circ}$ toe down bogie inclination angle. The unsteady CFD results have been used as an input to a FW-H solver to determine far field noise levels. The results show that strong vortices are generated when the flow separates from the front wheels. The interaction of these vortices with the solid landing gear surface is the main mechanism of noise generation. The results from the simulations show a clear relationship between the bogie inclination angle, the location of the strong vortex cores and the far field noise levels.
\end{abstract}

\section{Nomenclature}

$\begin{array}{ll}\text { ANDANTE } & \text { Aircraft Noise Disturbance Alleviation by Novel Technology } \\ \text { CFD } & \text { Computational Fluid Dynamics } \\ \text { DES } & \text { Detached Eddy Simulation } \\ \text { EPSRC } & \text { Engineering and Physical Sciences Research Council } \\ \text { FFT } & \text { Fast Fourier Transform } \\ \text { FW-H } & \text { Ffowcs Williams \& Hawkings } \\ \text { LES } & \text { Large Eddy Simulation } \\ \text { MLG } & \text { Main Landing Gear } \\ \text { PIV } & \text { Particle Image Velocimetry } \\ \text { RANS } & \text { Reynolds Averaged Navier Stokes } \\ \text { RMS } & \text { Root Mean Square } \\ \text { SA } & \text { Spalart Allmaras } \\ \text { SPL } & \text { Sound Pressure Level }\end{array}$

*PhD Student, School of Engineering Sciences, University of Southampton, SO17 1BJ, UK, Student Member AIAA.

† Senior Lecturer in Aeronautics, School of Engineering Sciences, University of Southampton, SO17 1BJ, UK, AIAA member.

${ }^{\ddagger}$ Research Fellow, School of Engineering Sciences, University of Southampton, SO17 1BJ, UK, AIAA member. 


$\begin{array}{lll}\text { symbol } & \text { unit } & \begin{array}{l}\text { description } \\ \alpha\end{array} \\ \rho & {\left[\frac{\mathrm{kg}}{\mathrm{m}^{3}}\right]} & \begin{array}{l}\text { inclination angle } \\ \text { density }\end{array} \\ C d & - & \text { drag coefficient } \\ C l & - & \text { lift coefficient } \\ C s & - & \text { side force coefficient } \\ D & {[\mathrm{~m}]} & \text { wheel diameter } \\ p_{R M S}^{\prime} & {[P a]} & \text { Root Mean Square of unsteady pressure } \\ p_{r e f} & {[P a]} & \text { reference pressure } \\ r & {[\mathrm{~m}]} & \text { distance } \\ r_{r e f} & {[\mathrm{~m}]} & \text { reference distance } \\ R e & - & \text { Reynolds number } \\ S t & - & \text { Strouhal number } \\ V & {\left[\frac{\mathrm{m}}{\mathrm{s}}\right]} & \text { free stream velocity } \\ y^{+} & - & \text {non-dimensional wall distance }\end{array}$

\section{Introduction}

$\mathrm{T}$ HE growth in the number of aircraft movements each year has led to pressure from the community living close to airports to reduce the noise generated by aircraft. Engine noise has been reduced significantly during the past decades due to the introduction of the high-bypass ratio turbo fan. With the increase in the size of aircraft, airframe noise has become just as important as engine noise during the approach phase. ${ }^{1}$

Airframe noise can be split into noise generated by the high lift devices and the landing gear. Flight tests have shown that for large wide-body aircraft landing gear noise is dominant over the noise of the high lift devices. ${ }^{1}$ Numerous experimental research has been performed to determine the major noise sources on the landing gear. ${ }^{2-4}$ Most of the experimental work focuses on the application of noise reduction treatments such as fairings. ${ }^{5-7}$ Fairings can be used to change the unsteady flow field around the landing gear and reduce the strength of the noise sources. Flow measurement techniques such as Particle Image Velocimetry (PIV) are difficult to apply to the flow around a main landing gear since the complex geometry often limits optical access. This makes it difficult to determine which flow features are responsible for the noise generation. Recent wind tunnel tests show that the bogie inclination angle has a strong influence on the noise radiation of a main landing gear. ${ }^{8}$

Improved turbulence models in Computational Fluid Dynamics (CFD) and the increase in computational power have

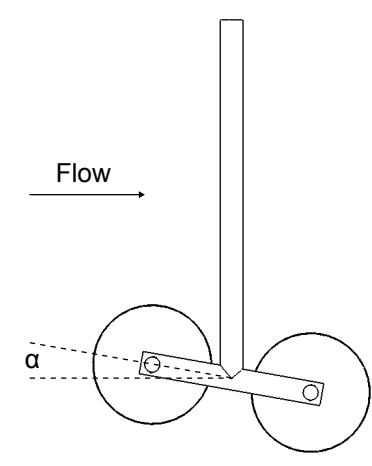

Figure 1. Bogie inclination angle $\alpha$. made it possible to do accurate simulations of the unsteady flow field around complex geometries such as landing gear. The data generated by CFD simulation can be used as source term in a noise radiation model to determine far field sound pressure levels. An advantage of CFD simulation over wind tunnel experiment is that it provides the full flow field. Most CFD simulations of the flow around landing gear have been done to compare different turbulence models and numerical solution techniques. ${ }^{9-12}$ Khorrami and Lockard $^{13}$ were $^{-1}$ the first to use CFD simulations in a landing gear noise reduction study.

In this paper Detached Eddy Simulation (DES) results will be presented of the flow field around a generic four wheel landing gear model with three different bogie inclination angles: $-10^{\circ}$ (toe down), $0^{\circ}$ (horizontal bogie) and $10^{\circ}$ (toe up), see Figure 1. A Ffowcs Williams and Hawkings (FW-H) solver has been used to determine far field noise levels and noise directivity of the three different landing gear configurations. 


\section{Methodology}

\section{II.A. Geometry}

The landing gear geometry is based on the model used by Li et al. ${ }^{6}$ for the ANDANTE (Aircraft Noise Disturbance Alleviation by Novel Technology) project. The geometry used in the ANDANTE project is a $1 / 4$ scale model based on the main A340 landing gear. This geometry is too complex for the current CFD study so it has been simplified. The focus of this research is the flow and noise from the bogie area so the upper part of the main landing gear is modeled by a clean strut. The lower part of the landing gear has been simplified by removing all small and medium sized parts and filling up the cavities. The resulting model consists of a main strut, bogie, axles and wheels (see Figure 2). Although this geometry is simplified it should give a good insight into the main flow features around a generic four wheel landing gear.

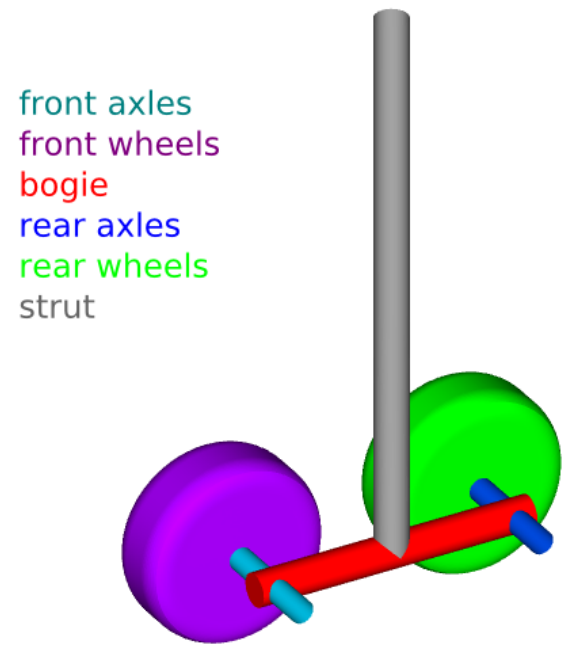

Figure 2. Generic main landing gear geometry (2 wheels removed for clarity).

\section{II.B. Grid Generation}

Grid generation is a compromise between accuracy and computational costs: a finer grid will result in a better resolved flow field but will also increase computational costs. Fully structured grids are preferred since they give a more accurate solution for the same number of cells compared to unstructured grids. Unfortunately, fully structured grids often lead to high cell density in the outer domain where a coarse grid would suffice. To avoid this problem a hybrid grid strategy was adopted since this type of grid can be easily matched with the grid requirements in the different regions of the domain.

The whole landing gear geometry is covered by a fully structured quadrilateral mesh. This mesh is extruded normal to the wall to form a layer of hexahedral cells. The cell next to the wall has a thickness to wheel diameter ratio of $1.39 \times 10^{-4}$ and is extruded using a growth rate of 1.1. This layer consists of 25 cells which captures the boundary layer and results in an $y^{+}$value of approximately 2 . The area around the bogie is the focus region of the grid and this whole area is filled with a structured grid with an average cell edge length of $5 \mathrm{~mm}\left(l / D=1.39 \times 10^{-2}\right)$. After filling up the space around the bogie and between the wheels a layer of structured cells has been wrapped around the whole landing gear which forms the end of the structured cells.

An unstructured grid of tetrahedral cells is used for the rest of the domain. A fine unstructured grid is used in the areas where the wake from the landing gear is expected. The outer region of the domain consists of a more coarse unstructured grid. The complete grid has approximately 8 million cells with 5.5 million structured cells close to the geometry and 2.5 million unstructured cells in the rest of the domain. 


\section{II.C. CFD setup}

The commercial software package Fluent (version 6.3.26) was used for the simulations. Fluent is based on the finite volume method and has various flow modelling capabilities which also includes a FW-H module. For this simulation the flow velocity is the same as used during the wind tunnel measurements by Li et al.: ${ }^{6} 40 \mathrm{~m} / \mathrm{s}$ which is approximately half the flight speed during the approach phase. The combination of the quarter scale model and reduced flow velocity leads to a Reynolds number of $9.9 \times 10^{5}$, based on the wheel diameter.

The turbulence model used for the simulation is Detached Eddy Simulation (DES) which combines a Reynolds Averaged Navier Stokes (RANS) turbulence model close to the walls and Large Eddy Simulation (LES) away from the walls. The Spalart Allmaras (SA) one equation turbulence model was chosen for the RANS region with standard model constants. This turbulence model switches to a sub grid model in the LES region. The boundary layer is treated as fully turbulent so that it resembles the flow around a full scale landing gear in flight condi-

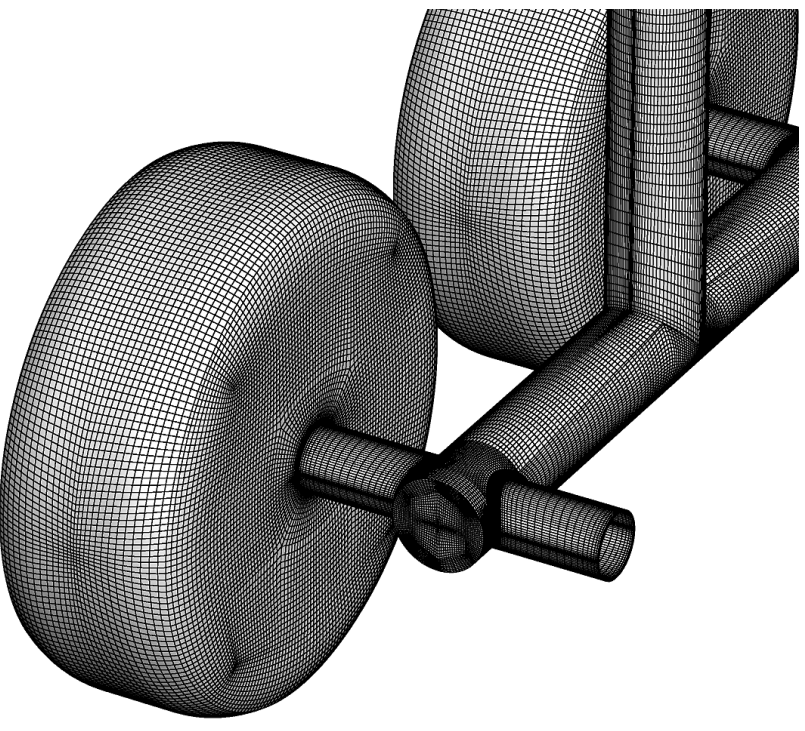

Figure 3. Detail of the surface mesh tions. A second order accurate solver in space and time was chosen for improved accuracy. A steady RANS solution has been computed and was used as initial flow field for the DES. Lift, drag and side force of the different landing gear components were monitored to ensure all transients have disappeared before data sampling was started.

\section{II.D. FW-H setup}

Fluents built in FW-H solver was used to calculate far field noise levels. Hedges et al. ${ }^{14}$ have shown that the strong wake behind the landing gear can cause problems with calculating far field noise from a permeable surface enclosing the volume sources. When a permeable FW-H surface is used a high quality grid is needed between the geometry and the permeable surface to make sure the acoustic waves are propagated properly.

To avoid the high density grid requirements and problems with the wake passing through the permeable surface the FW-H integration surface has been placed on the landing gear wall. This means that only the dipole sources are included in the far field noise. This is justified since for low Mach numbers the dipole sources are dominant over the quadrupole sources in the wake.

The FW-H surface on the landing gear wall is split into zones such that the far field noise contribution of the different landing gear components can

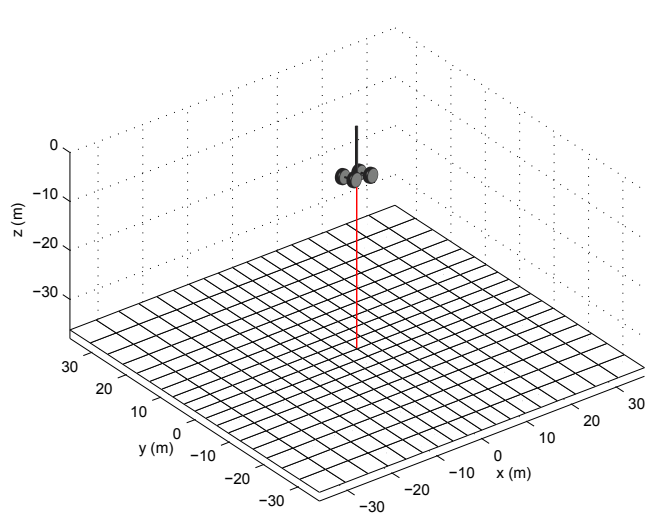

Figure 4. Far field noise directivity setup (landing gear is not to scale).

be determined. The far field noise level is calculated on a plane at a distance of 100 wheel diameters below the main landing gear such that the directivity of the noise can be shown (see Figure 4). 


\section{Results}

Flow data has been collected over a period of 9000 time steps during which the free stream flow has moved a distance of approximately 25 wheel diameters. The data has been used to determine mean and Root Mean Square (RMS) values of the flow variables. Noise spectra have been determined by splitting the data into 3 blocks of 4096 samples with an overlap of $50 \%$. Each block has been multiplied by a Hann windowing function before applying a Fast Fourier Transform (FFT). The Nyquist frequency of the resulting spectrum is $20 \mathrm{kHz}$ with a frequency resolution of $9.766 \mathrm{~Hz}$. The results of the three blocks have been averaged to determine the final spectrum.

\section{III.A. Aerodynamic Results}

\section{III.A.1. Forces}

The aerodynamic forces on the different landing gear components were monitored during the simulation. The aerodynamic coefficients have been determined by non dimensionalising these forces using the dynamic pressure $\left(1 / 2 \rho V^{2}\right)$ and a reference area. The size of the landing gear components differ considerably so therefor the center plane area of the wheel $\left(1 / 4 \pi D^{2}\right)$ has been used as the reference area.

The mean and RMS values of the lift coefficient of the various landing gear parts are given in Table 1. The strut has been omitted from this table since its lift force consists solely of shear forces which are relatively small. The largest variation in lift force between the different configurations is found on the front wheel and bogie. The location of flow separation on the front wheel depends strongly on the configuration. For a horizontal bogie the flow separation on the front wheel is symmetrical which results in a lift coefficient close to zero. The toe up configuration generates an asymmetric flow field around the front wheel with attached flow over the bottom and separated flow over the top of the wheel which results in a down force. The situation is reversed for the toe down configuration with separated flow at the bottom of the front wheel and attached flow over the top resulting in a lift force. There is a mean lift force on the horizontal bogie since the area where it is connected to the strut is not included in the force integration. The lift on the bogie behaves as expected: a positive inclination angle results in lift generation while a negative inclination angle leads to the generation of down force. The RMS values of the lift coefficient is small for the front wheel and front axle since they are in an undisturbed flow. Large fluctuations in lift coefficient are found for the bogie, rear wheel and rear axle since they are in the wake of the forward components.

\begin{tabular}{|c|c|l|c|l|l|l|}
\hline & \multicolumn{2}{|c|}{ horizontal $\left(\times 10^{-2}\right)$} & \multicolumn{2}{c|}{ toe up $\left(\times 10^{-2}\right)$} & \multicolumn{2}{c|}{ toe down $\left(\times 10^{-2}\right)$} \\
\hline component & Cl mean & Cl' rms & Cl mean & $\mathrm{Cl}^{\prime} \mathrm{rms}$ & $\mathrm{Cl}$ mean & $\mathrm{Cl}$ ' rms \\
\hline front wheel & 0.094 & 1.165 & -7.625 & 0.967 & 6.656 & 0.833 \\
rear wheel & -0.168 & 2.204 & -0.830 & 2.153 & -1.134 & 2.200 \\
front axle & 0.292 & 0.175 & -0.101 & 0.134 & 0.153 & 0.268 \\
rear axle & 0.379 & 0.962 & 0.455 & 1.051 & -0.158 & 0.843 \\
bogie & 1.104 & 2.904 & 4.242 & 0.910 & -5.063 & 0.753 \\
MLG & 4.674 & 4.890 & -10.790 & 5.638 & 6.312 & 3.697 \\
\hline
\end{tabular}

Table 1. Mean and RMS of the lift coefficient of the different MLG parts.

The mean and RMS values of the drag coefficient of the different components are listed in Table 2. The front and rear axle have not been included since their drag was a lot lower than the other components. The difference in drag between the three configurations is relatively small. The only clear trend from these results is that the rear wheel in each configuration has a slightly higher drag coefficient than the front wheel. The wake from the front wheel generates a relatively large area of near stagnation pressure on the rear wheel which creates the higher drag force. The drag coefficient of the bogie behaves as expected: it increases when the bogie is not aligned with the flow.

Table 3 shows the mean and RMS values of the side force coefficients of the different components except the front and rear axle since their side force consists purely of shear force and is very small. The front wheel in each configuration generates an outward side force due to the flow accelerating around the outside of the wheel. The flow velocity on the inside of the front wheel is a bit lower due to the blockage created by the bogie and strut. The rear wheel generates an inward side force for the horizontal configuration due to the flow 


\begin{tabular}{|c|c|l|c|l|c|l|}
\hline & \multicolumn{2}{|c|}{ horizontal $\left(\times 10^{-2}\right)$} & \multicolumn{2}{c|}{ toe up $\left(\times 10^{-2}\right)$} & \multicolumn{2}{c|}{ toe down $\left(\times 10^{-2}\right)$} \\
\hline component & Cd mean & Cd' rms & Cd mean & Cd' rms & Cd mean & Cd' rms \\
\hline front wheel & 16.734 & 1.692 & 15.257 & 1.393 & 15.420 & 1.426 \\
rear wheel & 19.980 & 1.760 & 16.384 & 1.397 & 16.483 & 1.538 \\
strut & 38.949 & 1.684 & 43.462 & 1.858 & 42.795 & 1.524 \\
bogie & 3.898 & 0.179 & 5.580 & 0.218 & 5.798 & 0.202 \\
MLG & 121.05 & 3.706 & 121.53 & 3.493 & 120.64 & 2.528 \\
\hline
\end{tabular}

Table 2. Mean and RMS of the drag coefficient of the different MLG parts.

accelerating between the wheel and the strut. The side force on the rear wheel for the other configurations is small. The strut shows very high side force fluctuations in all configurations due to the coherent vortex shedding at a frequency of $150 \mathrm{~Hz}$ which corresponds to a Strouhal number of approximately 0.23 . All three configurations are symmetric in the side force direction which should result in no mean side force for both the bogie and strut. The values are relatively low but not zero which indicates that the sampling period is too short due to limited computational resources.

\begin{tabular}{|c|l|r|l|r|r|l|}
\hline & \multicolumn{2}{|l|}{ horizontal $\left(\times 10^{-2}\right)$} & \multicolumn{2}{c|}{ toe up $\left(\times 10^{-2}\right)$} & \multicolumn{2}{c|}{ toe down $\left(\times 10^{-2}\right)$} \\
\hline component & Cs mean & Cs' rms & Cs mean & Cs' rms & Cs mean & Cs' rms \\
\hline front wheel & 12.214 & 2.343 & 8.139 & 1.832 & 6.663 & 1.236 \\
rear wheel & -3.763 & 3.291 & 0.448 & 3.076 & -0.208 & 2.752 \\
strut & -0.008 & 12.534 & 0.672 & 12.052 & 0.400 & 8.679 \\
bogie & -0.140 & 1.437 & 0.071 & 1.440 & -0.402 & 0.933 \\
MLG & -1.417 & 14.208 & 2.391 & 14.295 & 0.716 & 9.416 \\
\hline
\end{tabular}

Table 3. Mean and RMS of the side force coefficient of the different MLG parts.

\section{III.A.2. Flow features}

The unsteady CFD simulations generate large amounts of data which can be difficult to analyse. The amount of data can be reduced by studying the mean flow field however this leads to a loss of resolution since the weaker flow features get averaged out. A further reduction of data can be performed by using flow feature extraction techniques. An important flow feature from an aeroacoustic perspective are vortices and their interaction with solid surfaces. There are different methods to determine the location of the vortex core ${ }^{15}$ but the most popular ones are based on searching the domain for cells where the velocity vector is parallel to the vorticity vector. The mean velocity field has been used to determine the location of the vortex cores and stream lines have been started at the location of the vortex cores to indicate the rotation of the flow.

For the horizontal configuration there are four distinct vortex cores generated by the flow over each front wheel as can be seen in Figure 5. The two smaller vortices move outside of the rear wheels while the two larger vortices stay between the rear wheels parallel to the bogie. The vortex locations are based on the average flow field, in the unsteady flow the vortices will not be stationary but move around and generate strong fluctuations in pressure on the bogie and rear axle surface. Similar to the front wheel there are also four vortex cores behind the rear wheel which are all parallel to the free stream flow direction. Another vortex core is located behind the strut. The mean flow field shows a recirculation zone in this area but the unsteady results show coherent vortex shedding at a frequency of $150 \mathrm{~Hz}$.

The flow separates from the top of the front wheel in the toe up configuration creating two strong vortices as can be seen in Figure 6. The vortex from the outside of the front wheel moves over the top of the rear wheel. The vortex from the inside of the front wheel stays inside but near the top of the rear wheel. The presence of the two strong vortices near the top of the rear wheel will lead to large fluctuations in the surface pressure. The vortex cores behind the rear wheel and strut are similar to the horizontal configuration.

The flow field for the toe down configuration also shows two strong vortices behind the front wheel but in this case they pass below the rear wheel (Figure 7). The vortices are further away from the landing gear wall compared to the other two configurations which should result in lower level of unsteadiness in the surface pressure. The vortex core location behind the strut and rear wheel is similar to the other two configurations. 

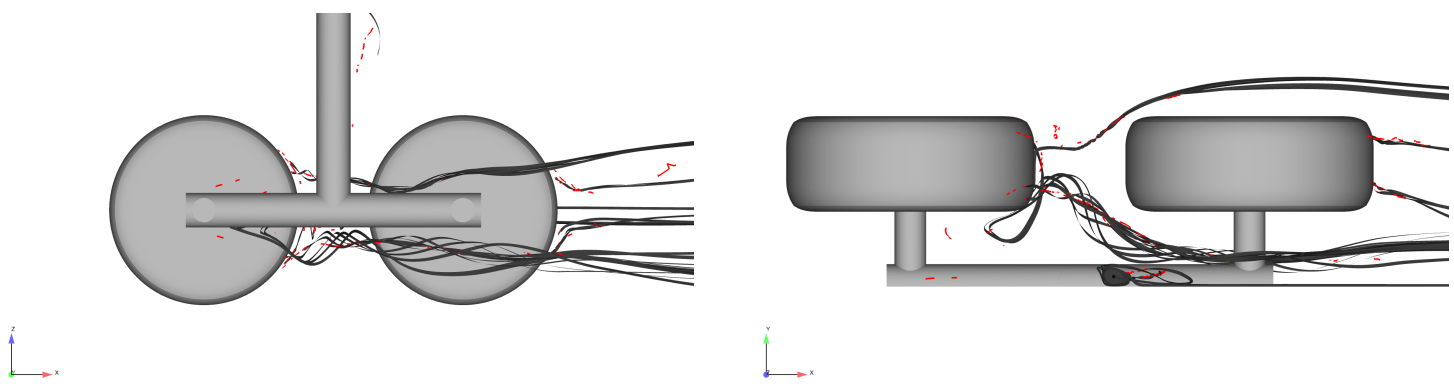

(a)

(b)

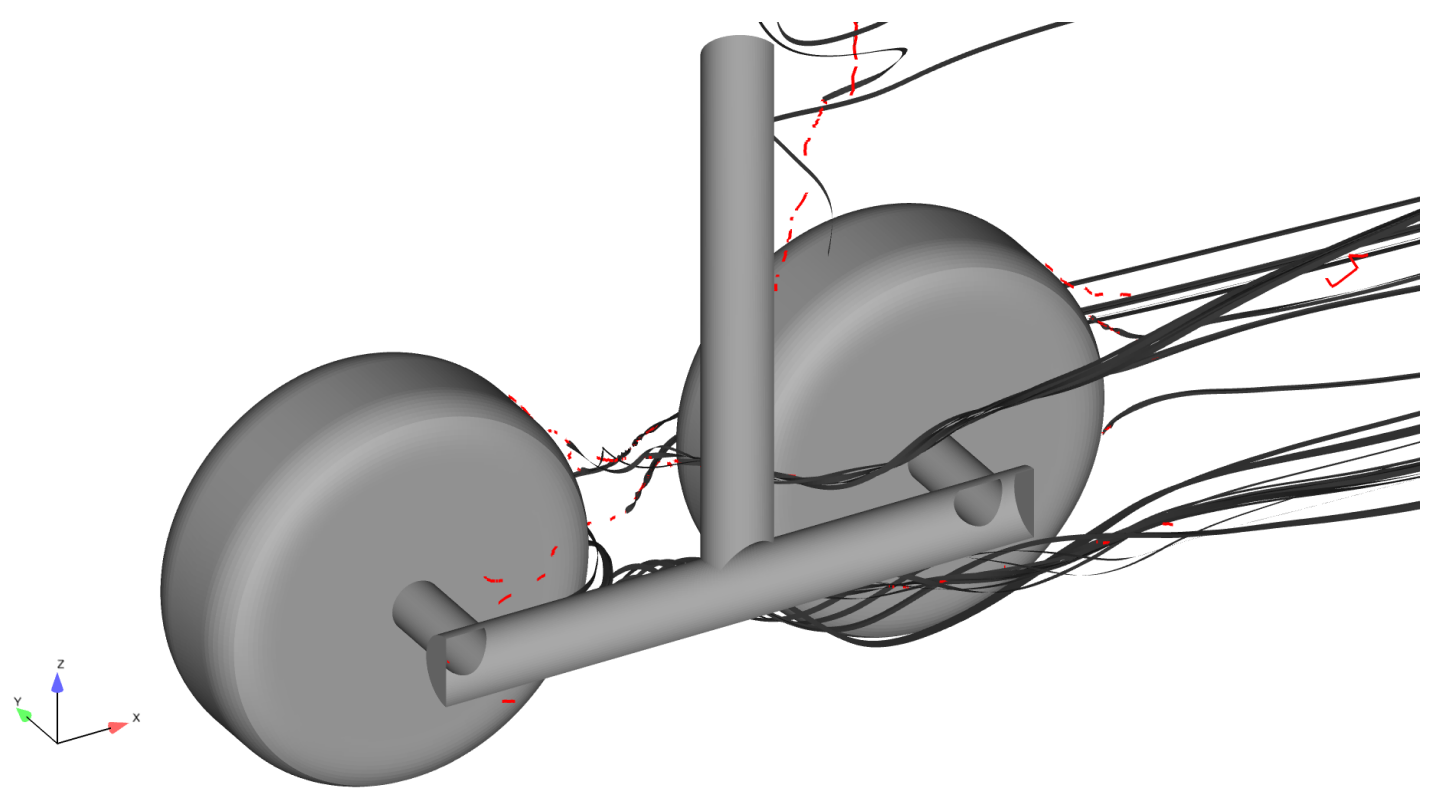

(c)

Figure 5. Side (a), top (b) and isometric view (c) of the vortex cores (red) and stream lines (black) around the horizontal landing gear configuration. 


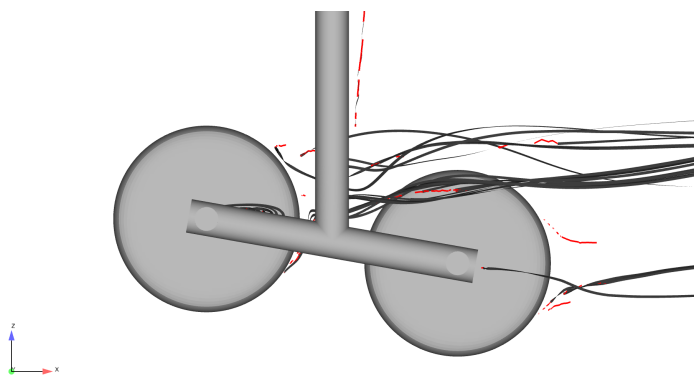

(a)

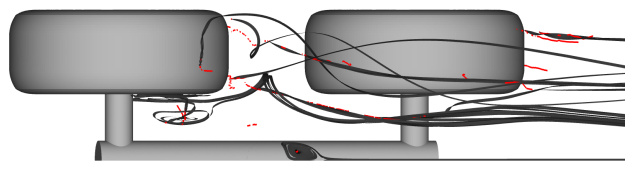

(b)

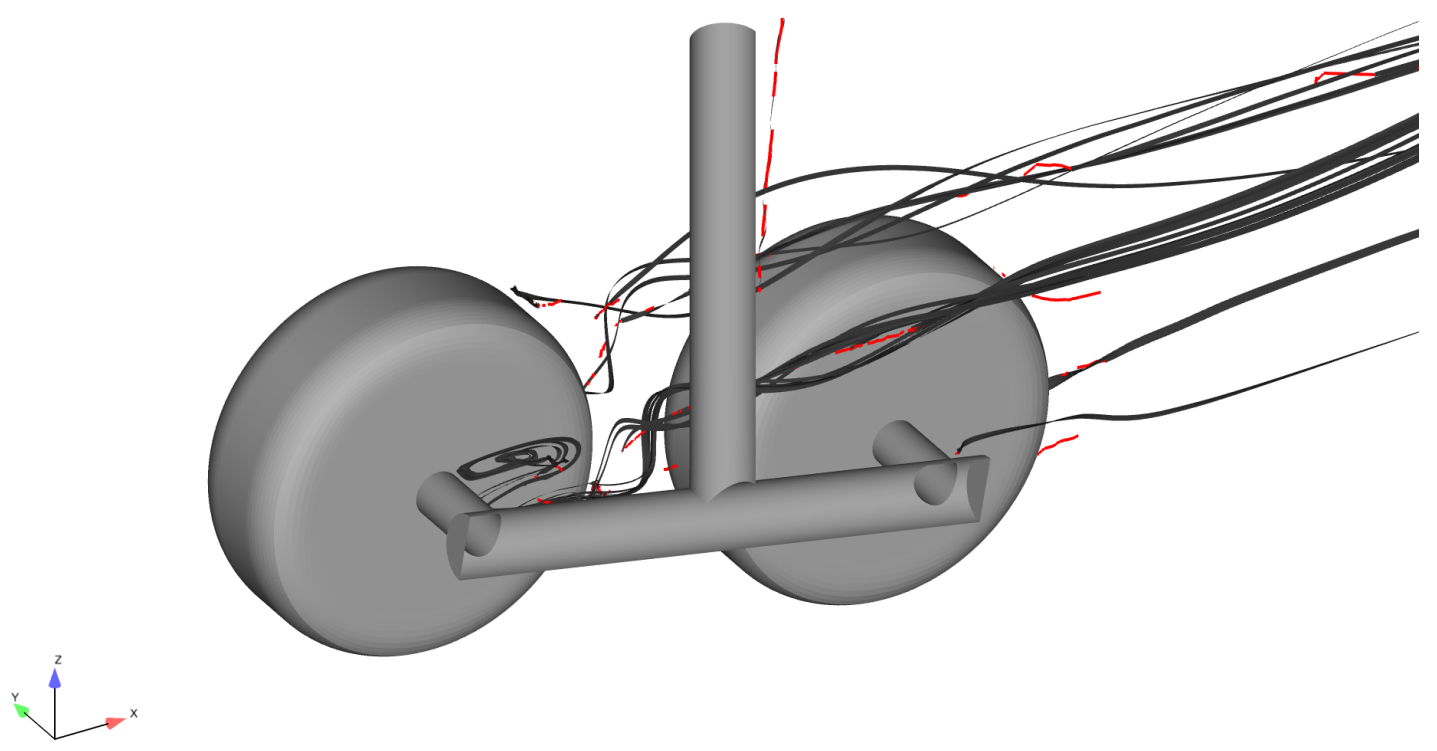

(c)

Figure 6. Side (a), top (b) and isometric view (c) of the vortex cores (red) and stream lines (black) around the toe up landing gear configuration. 


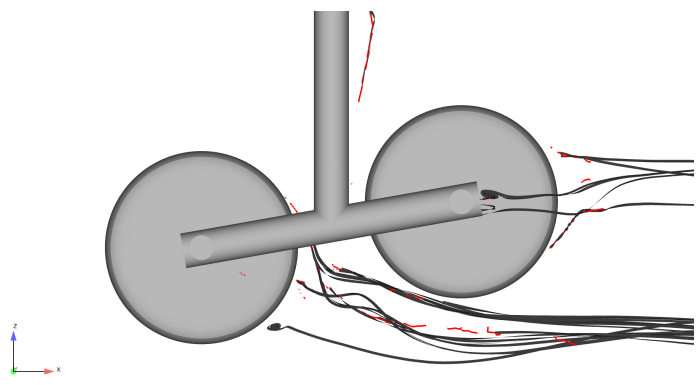

(a)

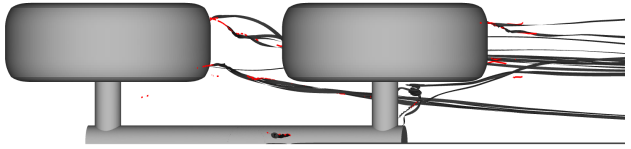

(b)

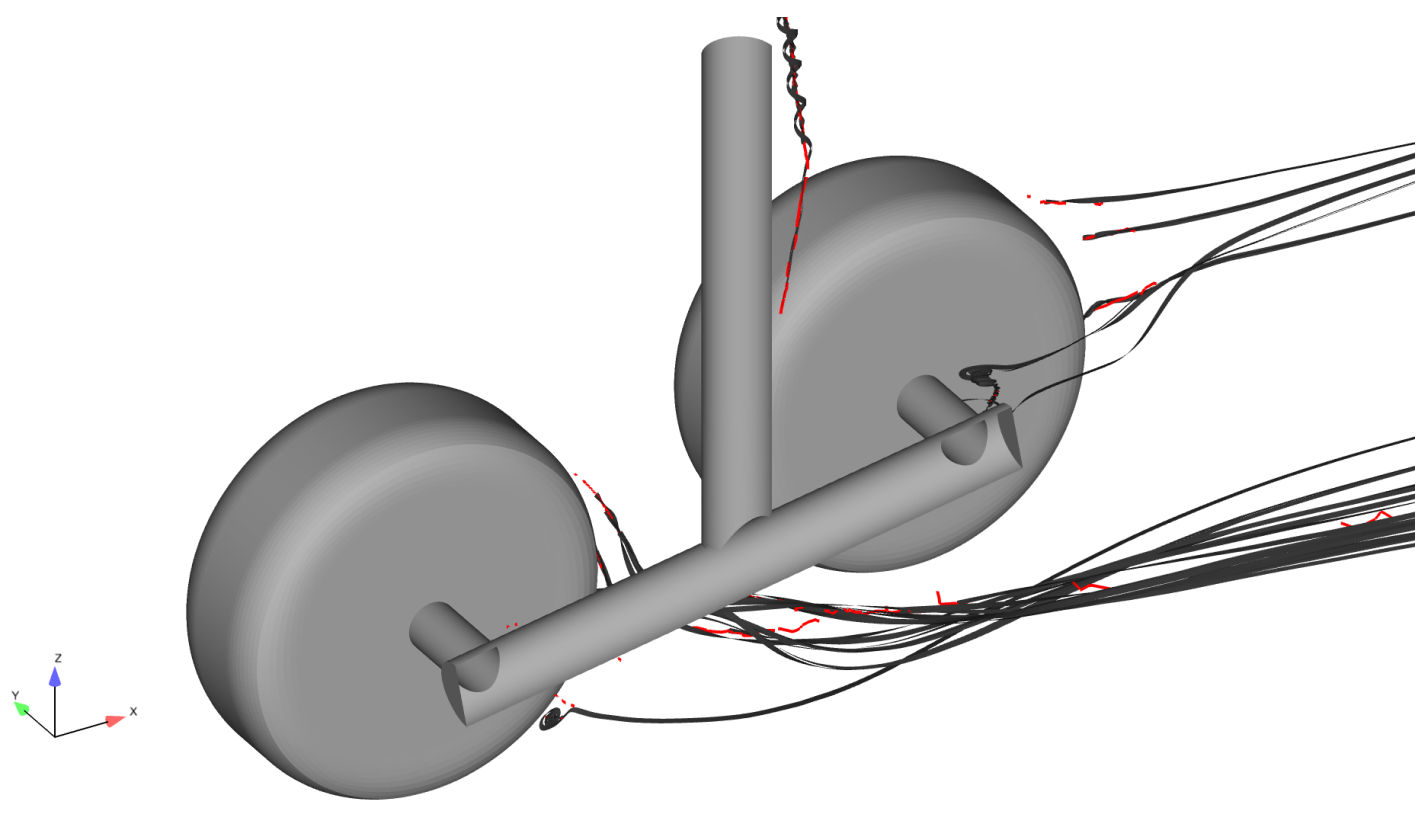

(c)

Figure 7. Side (a), top (b) and isometric view (c) of the vortex cores (red) and stream lines (black) around the toe down landing gear configuration. 


\section{III.B. Acoustic results}

This section will describe the noise spectra and noise directivity of the different landing gear configurations. The coherent vortex shedding behind the clean strut in all three configurations creates a strong dipole type source which dominates the far field noise levels. This makes it difficult to distinguish the noise contribution of the other landing gear components. The predominantly tonal noise from the main strut is not representative of a real landing gear since the presence of the door and other dressings leads to a disturbed flow field which prevents the formation of strong coherent vortices. The noise contribution of the clean strut has therefor not been included in the following sections.

\section{III.B.1. Noise levels}

The noise spectra of the three configurations (Figure 8, 9 and 10) show a haystack shape. The three landing gear configurations are very clean with no small scale details which lead to a quick drop off for higher frequencies noise levels. The bogie and the rear axles are the most important noise sources for the horizontal configuration 8. The flow feature extraction shows that the aft part of the bogie is surrounded by the vortices from the front wheels. The interaction of these large flow structures leads to a high level of low frequency noise. Further downstream these vortices interact with the rear axles which makes them the dominant mid and high frequency noise source. The front axles and front wheels are the least important noise sources since the flow that reaches these parts is undisturbed.

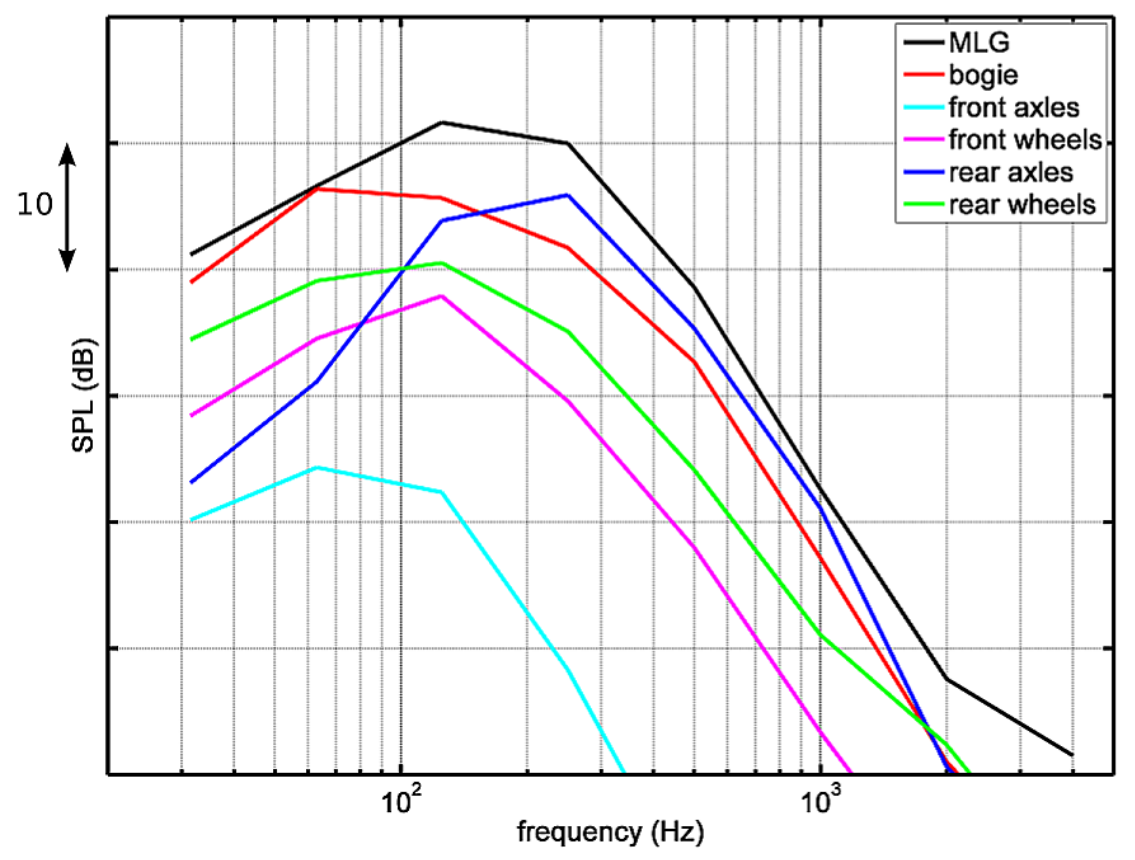

Figure 8. 1/3 octave band sound pressure levels generated by the different components of the horizontal landing gear configuration

The flow field for the toe up configuration is different from the horizontal one which results in a different noise level of the main landing gear and the components. The strong vortices from the front wheels interact with the rear wheels which makes the rear wheels the dominant low frequency noise source instead of the bogie. The turbulent wake flowing over the rear axles causes them to be the most important mid to high frequency noise source but the noise peak has shifted to a lower frequency. The change in vortex location has reduced the amount of unsteady flow around the bogie which leads to a large reduction of low frequency noise. The toe up configuration has a similar peak noise level as the horizontal configuration but the overall noise is less due to the large reduction of noise from the bogie.

The noise level generated by the toe down configuration is the lowest of all three configurations. The strong vortices from the front wheels interact with the rear wheels which causes them to be the dominant 


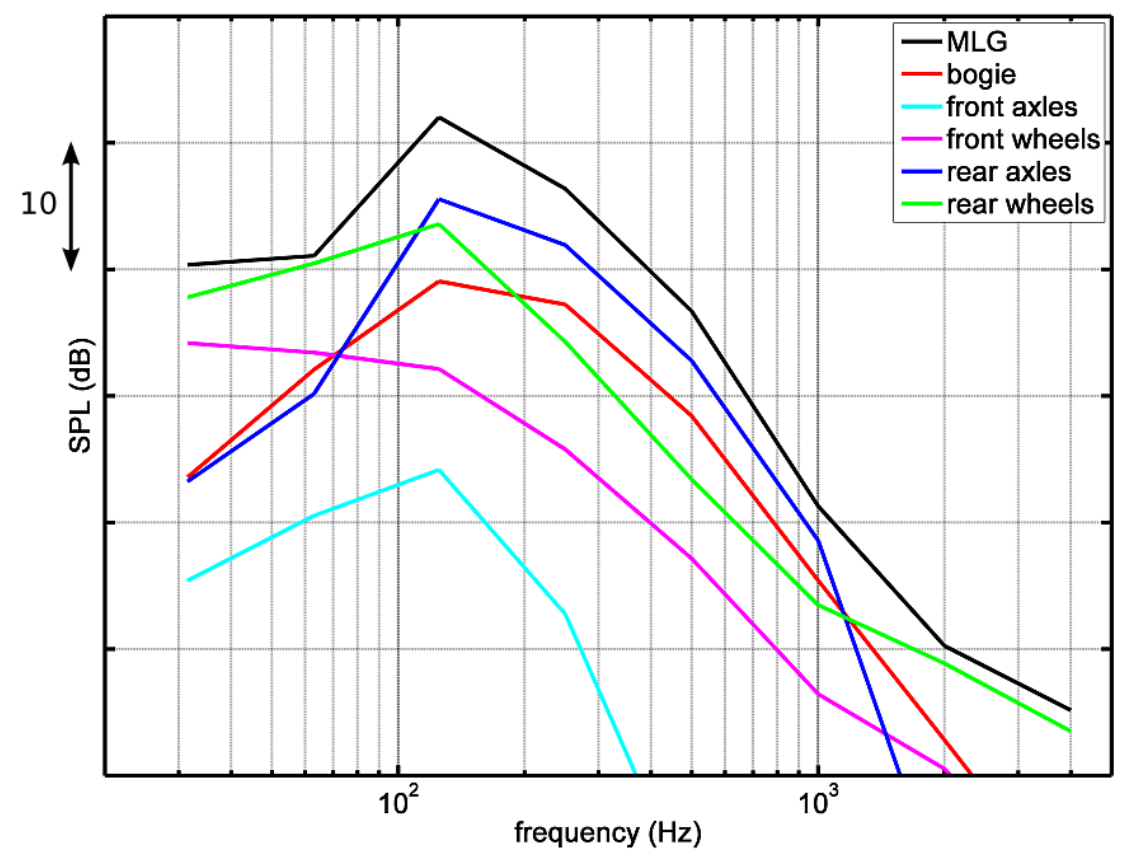

Figure 9. 1/3 octave band sound pressure levels generated by the different components of the toe up landing gear configuration

low noise source. The vortices are located at a greater distance from the landing gear than for the other two configurations which results in less unsteady flow over the rear axles and bogie and thus reduces the strength of these noise sources. The noise contribution from the front wheels has increased since there is a large area of separated flow on the bottom of the front wheels.

\section{III.B.2. Noise directivity}

The noise directivity of the three landing gear configurations has been determined by running the FW-H solver for 361 points on a horizontal plane 100 wheel diameters below the center of the landing gear. The total sound pressure level at each point has been determined according to the following equation:

$$
\mathrm{SPL}_{t o t}=20 \cdot \log \left\{\frac{p_{R M S}^{\prime}}{p_{\text {ref }}}\right\}
$$

where $p_{R M S}^{\prime}$ is the Root Mean Square of the acoustic pressure and $p_{r e f}$ the reference pressure of $2.0 \times 10^{-5}$ $\mathrm{Pa}$. The distance from the center of the landing gear to the points on the ground plane is not constant so the sound pressure levels need to be corrected for this. The following geometric correction has been applied to the calculated total sound pressure levels:

$$
\Delta \mathrm{SPL}=20 \cdot \log \left\{\frac{r}{r_{r e f}}\right\}
$$

where $r$ the distance from a point on the ground plane to the center of the landing gear is and $r_{r e f}$ the reference distance of 100 wheel diameters is. This correction is added to the total sound pressure level to get the corrected sound pressure level:

$$
\mathrm{SPL}_{c o r}=\mathrm{SPL}_{t o t}+\Delta \mathrm{SPL}
$$

The noise directivity of the three main landing gear configurations without the influence of the strut have been plotted in Figure 11. Low frequency pressure fluctuations contain most of the sound energy and thus contribute most to the total noise level. A solid surface will radiate most of the sound waves in the direction of the surface normal vector and the noise radiation will reduce quickly at other angles. The bogie is the 


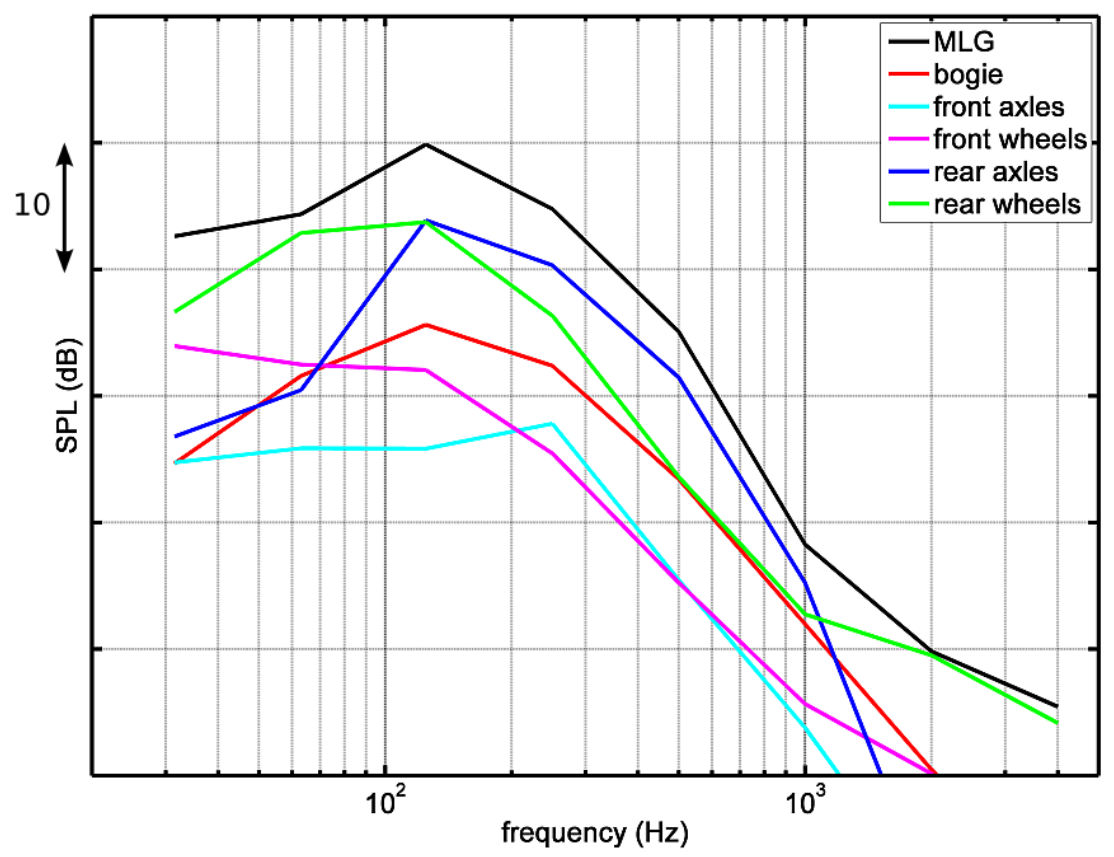

Figure 10. 1/3 octave band sound pressure levels generated by the different components of the toe down landing gear configuration

dominant noise source in the horizontal landing gear configuration and determines the shape of the noise contour of the whole landing gear. The orientation of the bogie surface normal vector results in most of the noise being radiated downwards and to the sides.

The noise directivity contour of the toe up configuration shows a clear peak directly below the landing gear. The rear axles and the rear wheels are the two strongest noise sources for this configuration. The rear axles radiate noise predominantly downwards while the large flat inside and outside surface area of the rear wheels lead to strong noise radiation to the sides. The rear axles are the dominant source over most of the frequency range so the shape of the total noise contour is similar to the noise contour of the rear axles.

The total noise contribution of the rear wheels and rear axles is similar for the toe down configuration, the rear wheels are the strongest low and high frequency noise source while the rear axles are dominant over the middle frequencies. This leads to a total noise contour for the toe down landing gear configuration which is a blend of the two individual contours. The noise peak below the landing gear caused by the rear axles combined with noise peaks to the sides generated by the rear wheels leads to a large oval shaped noise contour.

\section{Conclusion}

There is a strong relationship between the bogie inclination angle and the noise radiation of the simplified four wheel main landing gear. The relative position of the front and rear wheels determines the location of the flow separation on the front wheels which results in the formation of strong vortices. The interaction of these vortices with solid surfaces generates most of the low frequency noise. The vortices from the front wheels in the horizontal configuration are trapped between the rear wheels while they move downstream along the bogie and rear axles which makes this the noisiest main landing gear configuration. The toe down configuration is the quietest of the three since the strong vortices are shed from the bottom of the front wheels which leads to the largest distance between the vortices and solid surfaces. 


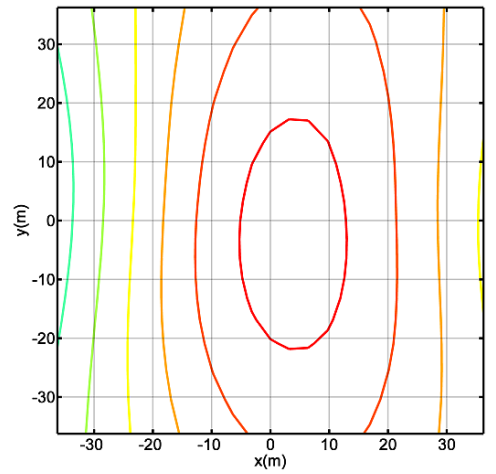

(a)

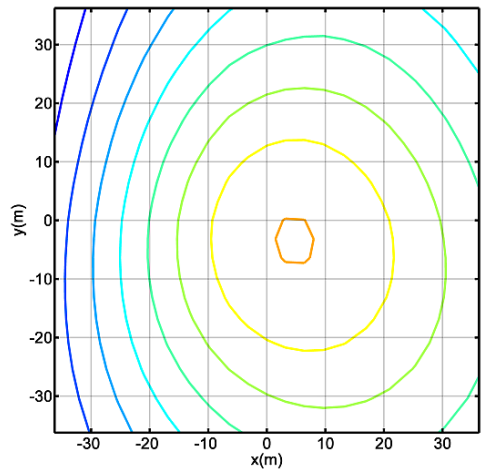

(b)

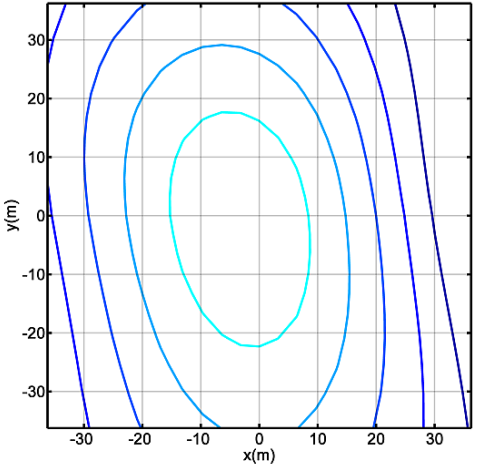

(c)

Figure 11. Corrected Sound Pressure Level contour of the horizontal (a), toe up (b) and toe down (c) landing gear configuration, $\Delta$ of $1 \mathrm{~dB}$ between contours, red indicates a high and blue a low level.

\section{Acknowledgements}

This work was supported by EPSRC (Engineering and Physical Sciences Research Council), the main government agency for funding research and training in engineering and the physical sciences in the United Kingdom. We would also like to acknowledge Stephen Chow and Nicolas Molin of Airbus and professor Xin Zhang of Southampton University for helpful discussions.

\section{References}

${ }^{1}$ Chow, L., Mau, K., and Remy, H., "Landing gears and high lift devices airframe noise research," 8th AIAA/CEAS Aeroacoustics Conference and Exhibit, 2002.

${ }^{2}$ Jaeger, S., Burnside, N., Soderman, P., Home, W., and James, K., "Microphone Array Assessment of an Isolated 26\%Scale, High-Fidelity Landing Gear," 8th AIAA/CEAS Aeroacoustics Conference and Exhibit, Vol. 2410, 2002.

${ }^{3}$ Ravetta, P., Burdisso, R., and Ng, W., "Wind Tunnel Aeroacoustic Measurements of a 26\%-scale 777 Main Landing Gear Model," 10th AIAA/CEAS Aeroacoustics Conference, Vol. 2885, 2004.

${ }^{4}$ Remillieux, M., Camargo, H., Burdisso, R., and Ng, W., "Aeroacoustic Study of a $26 \%$-Scale, High-Fidelity, Boeing 777 Main Landing Gear in a Semi-Anechoic-Wind-Tunnel Test Section," 13th AIAA/CEAS Aeroacoustics Conference (28th AIAA Aeroacoustics Conference), 2007.

${ }^{5}$ Dobrzynski, W., Schöning, B., Chow, L., Wood, C., Smith, M., and Seror, C., "Design and testing of low noise landing gears," International Journal of Aeroacoustics, Vol. 5, No. 3, 2006, pp. 233-262.

${ }^{6} \mathrm{Li}$, Y., Smith, M., Zhang, X., and Molin, N., "Noise Sources Control of an Aircraft Landing Gear," 13th AIAA/CEAS Aeroacoustics Conference (28th AIAA Aeroacoustics Conference), 2007.

${ }^{7}$ Ravetta, P., Burdisso, R., and Ng, W., "Noise Control Of Landing Gears Using Elastic Membrane-Based Fairings," 13th AIAA/CEAS Aeroacoustics Conference (28th AIAA Aeroacoustics Conference), 2007.

${ }^{8}$ Dobrzynski, W., Chow, L., Smith, M., Boillot, A., Dereure, O., and Molin, N., "Experimental Assessment of Low Noise Landing Gear Component Design," 15th AIAA/CEAS Aeroacoustics Conference, Miami, 2009.

${ }^{9}$ Souliez, F., Long, L., Morris, P., and Sharma, A., "Landing gear aerodynamic noise prediction using unstructured grids," International Journal of Aeroacoustics, Vol. 1, No. 2, 2002, pp. 115-135.

${ }^{10}$ Lockard, D., Khorrami, M., and Li, F., "Aeroacoustic analysis of a simplified landing gear," 9th AIAA/CEAS Aeroacoustics Conference and Exhibit, 2003.

${ }^{11}$ Lockard, D., Khorrami, M., and Li, F., "High Resolution Calculation of a Simplified Landing Gear," 10th AIAA/CEAS Aeroacoustics Conference, 2004.

${ }^{12}$ Seror, C., Sagaut, P., and Belanger, A., "A Numerical Aeroacoustic Analysis of a Detailed Landing Gear," 10th AIAA/CEAS Aeroacoustics Conference, 2004.

${ }^{13}$ Khorrami, M. and Lockard, D., "A Landing Gear Noise Reduction Study Based on Computational Simulations," InterNoise 2006, 2006.

${ }^{14}$ Hedges, L., Travin, A., and Spalart, P., "Detached-Eddy Simulations Over a Simplified Landing Gear," Journal of Fluids Engineering, Vol. 124, 2002, pp. 413.

${ }^{15}$ Jiang, M., Machiraju, R., and Thompson, D., The Visualization Handbook, Elsevier Academic Press, 2004. 